\title{
Aplicación de prácticas en gerencia de proyectos de investigación, desarrollo tecnológico e innovación en grupos de investigación
}

DOI: https://doi.org/10.21158/01208160.n90.2021.2974

\author{
Diana Carolina Contreras-Gutiérrez ${ }^{1}$ \\ Institución Universitaria CEDOC \\ dianacontrerasgutierrez@cedoc.edu.co \\ Nelson de Jesús Moreno-Ávila² \\ Escuela Colombiana de Ingeniería Julio Garavito \\ ndmorenoa@unal.edu.co \\ Gloria Viviana Pérez-Londoño ${ }^{3}$ \\ Escuela Colombiana de Ingeniería Julio Garavito \\ gloria.perez-1@mail.escuelaing.edu.co \\ César Augusto Leal-Coronado ${ }^{4}$ \\ Escuela Colombiana de Ingeniería Julio Garavito \\ cesar.leal@escuelaing.edu.co
}

Fecha de recepción: 02 de noviembre de 2020

Fecha de aprobación: 27 de mayo de 2021

Fecha de publicación: 05 de agosto de 2021

Cómo citar este artículo / To reference this article / Comment citer cet article / Para citar este artigo:

Contreras-Gutiérrez, D. C.; Moreno-Ávila, N.; Pérez-Londoño, G. V.; Leal-Coronado, C.A. (2021). Aplicación de prácticas en gerencia de proyectos de investigación, desarrollo tecnológico e innovación en grupos de investigación. Revista Escuela de Administración de Negocios, (90), 47-64. DOI: https://doi.org/10.21158/01208160.n90.2021.2974

\section{Resumen}

Esta investigación tuvo como objetivo identificar los procesos gerenciales que realizan los investigadores para la planeación, ejecución y seguimiento de los proyectos de investigación, desarrollo tecnológico e innovación, con el fin de categorizar por niveles la aplicación de prácticas en gerencia de proyectos por parte de los grupos de investigación. El estudio se enfoca en generar un método de valoración de prácticas gerenciales, aplicable a la gestión de proyectos de $\mathrm{I}+\mathrm{D}+\mathrm{i}$ y dirigido a organizaciones dedicadas al desarrollo de procesos de ciencia y tecnología. Para esto se creó un instrumento que tiene como propósito identificar y medir las prácticas en gerencia, el cual se aplicó en una muestra de 329 grupos de investigación ubicados

1 Administradora Ambiental - Universidad Distrital Francisco José de Caldas. Especialización en formulación y evaluación social y económica de proyectos - Universidad Católica de Colombia. Maestría en desarrollo y gerencia integral de proyectos - Escuela Colombiana de Ingeniería Julio Garavito. ORCID: https://orcid.org/0000-0002-8996-5348

2 Ingeniero Agrícola - Universidad Nacional. Ingeniero Civil - Universidad Agraria de Colombia. Maestría en desarrollo y gerencia integral de proyectos - Escuela Colombiana de Ingeniería Julio Garavito. ORCID: https://orcid.org/0000-0003-0120-9212

3 Tecnóloga en mecánica industrial - Universidad Tecnológica de Pereira. Ingeniera Civil - Universidad La Gran Colombia. Maestría en desarrollo y gerencia integral de proyectos - Escuela Colombiana de Ingeniería Julio Garavito. ORCID: https://orcid.org/00000002-6639-3981

4 Ingeniero Civil - Escuela Colombiana de Ingeniería Julio Garavito. Especialización en recursos hidráulicos y medio ambiente Escuela Colombiana de Ingeniería Julio Garavito. Master of engineering in construction engineering and management - University of Michigan, Ann Arbor. ORCID: https://orcid.org/0000-0002-6306-8876 
en Bogotá. Se diseñó un cuestionario para las tres áreas de conocimiento que conforman la triple restricción en la gerencia de proyectos: alcance, cronograma y costo, así como sus respectivos procesos. Entre los resultados obtenidos se identificó que los grupos aplican procesos gerenciales empíricos producto de su experiencia, al categorizar el 74,1 \% de los grupos en un nivel medio de aplicación de prácticas de gerencia y resaltar que los procesos más críticos para los investigadores son la validación del alcance y la determinación del presupuesto.

Palabras clave: gerencia de proyectos; procesos gerenciales; prácticas gerenciales; grupos de investigación; proyectos de investigación; investigación, desarrollo tecnológico e innovación; procesos de ciencia y tecnología.

\title{
Application of research, technological development and innovation project management practices in research groups
}

\begin{abstract}
This research aims at identifying the management processes that researchers must complete for the planning, execution, and follow-up of Research, Technological Development, and Innovation (R\&D\&I) projects, in order to categorize -by levels- the application of project management practices on the part of research groups. The study focuses on generating a method for evaluating management practices, applicable to the management of R\&D\&I projects and aimed at organizations that are dedicated to the development of science and technology processes. For this purpose, we created an instrument to identify and measure management practices, which was applied to a sample of 329 research groups located in Bogota. The questionnaire was designed for the three areas of knowledge that make up the triple constraint in project management: scope, schedule, and cost, as well as their respective processes. Among the results achieved, we identified that the groups apply empirical management processes as a result of their experience, categorizing $74,1 \%$ of the groups at a medium level of application of management practices and highlighting that the most critical processes for the researchers are the validation of the scope and the determination of the budget.
\end{abstract}

Keywords: project management; management processes; management practices; research groups; research projects; research, technological development and innovation; science and technology processes.

\section{$\longrightarrow$ Aplicação de práticas em gestão de projetos de pesquisa, desenvolvimento tecnológico e inovação em grupos de pesquisa}

\section{Resumo}

O objetivo desta pesquisa foi identificar os processos gerenciais realizados por pesquisadores para o planejamento, execução e acompanhamento de projetos de pesquisa, desenvolvimento tecnológico e inovação, a fim de categorizar, por níveis, a aplicação das práticas de gestão de projetos pelos grupos de pesquisa. $\mathrm{O}$ estudo centraliza-se em gerar um método de avaliação de práticas de gestão, aplicável à gestão de projetos de $\mathrm{P}+\mathrm{D}+\mathrm{i}$ e voltado para organizações que se dedicam ao desenvolvimento de processos de ciência e tecnologia. Para isso, foi criado um instrumento cujo objetivo é identificar e mensurar práticas de gestão, o qual foi aplicado em uma amostra de 329 grupos de pesquisa localizados em Bogotá. Foi elaborado 
um questionário para as três áreas de conhecimento que compõem a tripla restrição em gestão de projetos: escopo, cronograma e custo, bem como seus respectivos processos. Dentre os resultados obtidos, identificouse que os grupos aplicam processos de gestão empíricos a partir de sua vivência, categorizando $74,1 \%$ dos grupos em nível médio de aplicação de práticas de gestão e destacando que os processos mais críticos para os pesquisadores são a validação de escopo e a determinação do orçamento.

Palavras-chave: gerenciamento de projetos; processos gerenciais; práticas de gestão; grupos de pesquisa; projetos de pesquisa; pesquisa, desenvolvimento tecnológico e inovação; processos de ciência e tecnologia.

\section{Mise en place des pratiques de gestion de projets, développement technologique et innovation dans les groupes de recherche}

\section{Résumé}

L'objectif de cette étude consiste à identifier les processus managériaux menés par les chercheurs pour la planification, l'exécution et le suivi des projets de recherche, de développement technologique et d'innovation, afin de catégoriser l'application des pratiques de gestion de projet par niveaux. L'étude se concentre sur la mise en place d'une méthode d'évaluation des pratiques managériales, applicable à la gestion de projets de $\mathrm{R} \& \mathrm{D}+\mathrm{i}$ et destinée aux entreprises axées sur le développement de processus scientifiques et technologiques. Un instrument spécifique a ainsi été créé pour identifier et mesurer les pratiques de gestion, et qui a été appliqué sur un échantillon de 329 groupes de recherche situés à Bogotá. Un questionnaire a été conçu pour les trois domaines de compétences composant la triple restriction de la gestion de projet: portée, échéancier et coût, et leurs processus respectifs. Les résultats obtenus montrent que les groupes de recherche appliquent des processus de gestion empiriques en fonction de leur expérience, avec 74,1\% d'entre eux pratiquant un niveau intermédiaire d'application des modes de gestion et montrant que les processus critiques pour les chercheurs sont la validation de la portée et la détermination du budget.

Mots-clés: gestion de projet; processus de gestion; pratiques managériales; groupes d'investigation; projets de recherche; recherche, développement technologique et innovation; processus scientifiques et technologiques. 


\section{Introducción}

$\mathrm{E}^{1}$ objetivo de esta investigación es diseñar un método que permita identificar y valorar la aplicación de prácticas en gerencia de proyectos en los grupos de investigación. Para esto se elaboró un instrumento con el fin de recopilar la información de los grupos de investigación sobre gerencia de proyectos basados en el Project Management Institute (PMI). Luego, se diseñó el método de valoración con el fin de medir la aplicación de prácticas en gerencia; esta medición se realizó mediante el indicador de nivel de gerencia (ING). Después de aplicar el instrumento y valorar las prácticas en gerencia de proyectos, se obtuvo una clasificación de los grupos de investigación según su ING.

En la sustentación teórica del trabajo se tratan conceptos de ciencia, tecnología e innovación (CTI), los cuales han constituido un motor indiscutible de crecimiento y desarrollo en los países (Bortagaray, 2016). La CTI la integran actividades científicas y tecnológicas (ACT) que comprenden la enseñanza y la formación científica y técnica (STET), los servicios científicos y técnicos (SCT) y la investigación, el desarrollo tecnológico y la innovación (Organización para la Cooperación y el Desarrollo Económicos [OCDE], 2018).

Los grupos de investigación contribuyen al desarrollo de la CTI, al ser el conjunto de personas con diferentes roles que aportan de forma directa a las actividades de investigación, desarrollo tecnológico e innovación I+D+i (OCDE, 2018). En Colombia, los grupos con capacidades en CTI son los responsables de la formulación y ejecución de proyectos de $\mathrm{I}+\mathrm{D}+\mathrm{i}$ y tienen como fin aportar al nuevo conocimiento mediante la generación de productos científicos. Los grupos se han sometido a procesos de medición por parte de Minciencias, de modo que se clasifican en diferentes categorías, dependiendo, principalmente, de la producción científica.
La I+D+i hace parte del funcionamiento de CTI y comprende cuatro actividades: investigación básica, investigación aplicada, desarrollo experimental e innovación (OCDE, 2018). El manual de Frascati y Oslo define cada una de estas actividades como se refiere en los siguientes puntos.

- Investigación básica: trabajos experimentales o teóricos para obtener nuevos conocimientos (OCDE, 2018).

- Investigación aplicada: trabajos originales para adquirir nuevos conocimientos, dirigidos hacia un objetivo práctico (OCDE, 2018).

- Desarrollo experimental: trabajos sistemáticos que aprovechan los conocimientos obtenidos de la investigación o experiencia práctica con miras a la producción de nuevos materiales, productos o dispositivos (OCDE, 2018).

- Innovación: actividades encaminadas al desarrollo de nuevos productos, lo que incluye fases del desarrollo de preproducción, la producción y la distribución (OCDE, 2007).

$\mathrm{La}+\mathrm{D}+\mathrm{i}$ se realiza a través de proyectos, definidos como el conjunto temporal de actividades con altas incertidumbres; son complejos y están destinados a cumplir con el descubrimiento científico y la producción de nuevos conocimientos (Forozandeh, Teimoury y Makui, 2019; Morandi, 2013). Youker (2002) argumenta que este tipo de proyectos suelen ser de largo plazo, de manera que la calidad tiene prioridad sobre el tiempo, dado que es un proceso intelectual. De acuerdo con Kuchta y Skowron (2015), estos proyectos no tienen directrices uniformes en gerencia de proyectos. Es así como Colciencias (2013) identificó que existe una baja función en el seguimiento de proyectos y sugiere profesionalizar la gerencia en proyectos de $\mathrm{I}+\mathrm{D}+\mathrm{i}$. 
Para Goldstein y Kearney (2020), los investigadores con periodicidad modifican la frecuencia de los proyectos, especialmente los plazos, de manera que estos son cambios sensibles en el buen rendimiento. También, se encuentra que estos proyectos tienen una línea de tiempo corta y un presupuesto reducido, de modo que obtienen menos probabilidades de generar resultados a corto plazo y es evidente la necesidad de aplicar gerencia de proyectos con el fin de mejorar la productividad y la financiación.

Kuchta, et al. (2017) afirman que la falta de aplicación de prácticas de gerencia de proyectos produce informes de gestión académica poco confiables que, con frecuencia, se preparan para cumplir criterios y no son un reflejo fiable del desempeño del proyecto. Por tanto, Martínez y Páez (2017) señalan que la evaluación de los resultados de investigación y los impactos requieren una comprensión de los objetivos del proyecto. En este sentido, Cassanelli, Guiridlian-Guarino y Fernández-Sánchez (2014) proponen caracterizar los proyectos de $\mathrm{I}+\mathrm{D}+\mathrm{i}$ en un espectro amplio y establecer herramientas en gerencia de proyectos.

Para Youker (2002), esta gerencia de proyectos de investigación y desarrollo tecnológico debe ser flexible por su larga duración y es esencial en el propósito de establecer los objetivos y medir el progreso contra ellos. Bark, Kragt y Robson (2016) proponen cuatro buenas prácticas de gerencia en proyectos de $\mathrm{I}+\mathrm{D}+\mathrm{i}$ : a) desarrollar un modelo conceptual, b) apoyar la comunicación, c) establecer una revisión independiente, y d) un proceso de síntesis. En conclusión, la gestión activa en proyectos de investigación permite la mitigación de riesgos (Goldstein y Kearney, 2020).

Por otra parte, diferentes organizaciones se han dedicado al desarrollo de proyectos de investigación, tanto instituciones académicas como el sector empresarial e instituciones del Estado (Huang y Jong, 2019), de manera que se ha aumentado la inversión y las alianzas de triple y cuádruple hélice para la investigación y la innovación (Brem y Radziwon, 2017; Mêgnigbêto, 2018; Sarpong et al., 2017).

En un aspecto más técnico en gerencia de proyectos, para el Project Management Institute (2017) esta es la aplicación de conocimientos, habilidades, herramientas y técnicas a las actividades necesarias para alcanzar los objetivos del proyecto. De este modo, el PMI es de gran interés para las organizaciones de todos los sectores (Montes-Guerra, Gimena-Ramos y Díez-Silva, 2013).

La guía Project management body of knowledge (PMBOK) es un estándar en gerencia de proyectos del PMI en la que se encuentran los lineamientos, las herramientas, las técnicas, los procesos y las áreas dirigidas a la aplicación de gerencia de proyectos. Emitida por el Project Management Institute, incluye prácticas tradicionales comprobadas y ampliamente utilizadas, así como prácticas innovadoras emergentes para la profesión. En su sexta edición también incluye las prácticas recapituladas en procesos. Cada proceso produce una o más salidas a partir de una o más entradas mediante el uso de herramientas y técnicas adecuadas. La organización de procesos se hace de manera lógica y se agrupan en cinco grupos de procesos, a la vez que estos se categorizan en diez áreas de conocimiento (Project Management Institute, 2017).

El PMI es el estándar de mayor difusión, al ser el más utilizado para la elaboración de metodologías y certificación (Montes-Guerra et al., 2013). Youker y Kähkönen (como se citan en Sánchez-Serna, Martínez y Del Río-Cortina, 2017), afirman que el PMI plantea un estándar aplicable a cualquier tipología de proyectos. Deriva, además, una serie de herramientas que de fondo son adaptables, fáciles de implementar, no requieren software especializado y permiten llevar un proyecto a su buen término (Millán, 2017). 
En el PMI se definen los proyectos como un esfuerzo temporal, con un entregable único - un producto, servicio o resultado-, el cual debe tener un principio y un fin definidos (Project Management Institute, 2017). Una inadecuada gerencia de proyectos puede conducir al incumplimiento de plazos, sobrecostos, calidad deficiente, retrabajo, expansión no controlada del proyecto, pérdida de reputación para la organización, interesados insatisfechos e incumplimiento de los objetivos propuestos (Project Management Institute, 2017).

Para el estudio de la gerencia de proyectos mediante el PMI es común abordar los temas por grupos de proceso y áreas de conocimiento, involucrando una serie de entradas, herramientas y resultados (Montes-Guerra et al., 2013).
En gerencia de proyectos los modelos de madurez permiten medir la capacidad de las organizaciones de gestionar sus proyectos exitosamente (SolartePazos y Sánchez-Arias, 2014). Los modelos de madurez existentes se basan en la aplicación y utilización de terminología planteada en cada uno de los estándares correspondientes propuestos; al aplicar un modelo de madurez en una organización dedicada al desarrollo de la investigación, se obtiene como resultado que la organización se ubica en los niveles más bajos del modelo (Cassaneli et al., 2009).

\section{Metodología}

耳 1 diseño metodológico de la investigación es de tipo cuantitativo y alcance descriptivo, busca identificar

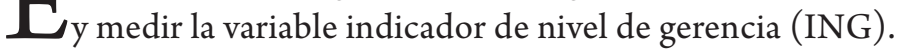

\subsection{Diseño del instrumento}

Para el diseño del instrumento se definen los componentes como las áreas del conocimiento que propone el PMI. A fin de seleccionar estos componentes se determinan las áreas de conocimiento que se consideran como restricciones en la gerencia de proyectos — alcance, cronograma y costo-. Las restricciones son limitantes en la ejecución de los proyectos (Lledo y Rivarola, 2007; Mulcahy, 2013).

Se diseñó un cuestionario para las tres áreas de conocimiento que conforman la triple restricción: alcance, cronograma y costo, así como sus respectivos procesos (véase la Figura 1). 
Figura 1. Áreas de conocimiento y procesos propuestos por el PMI

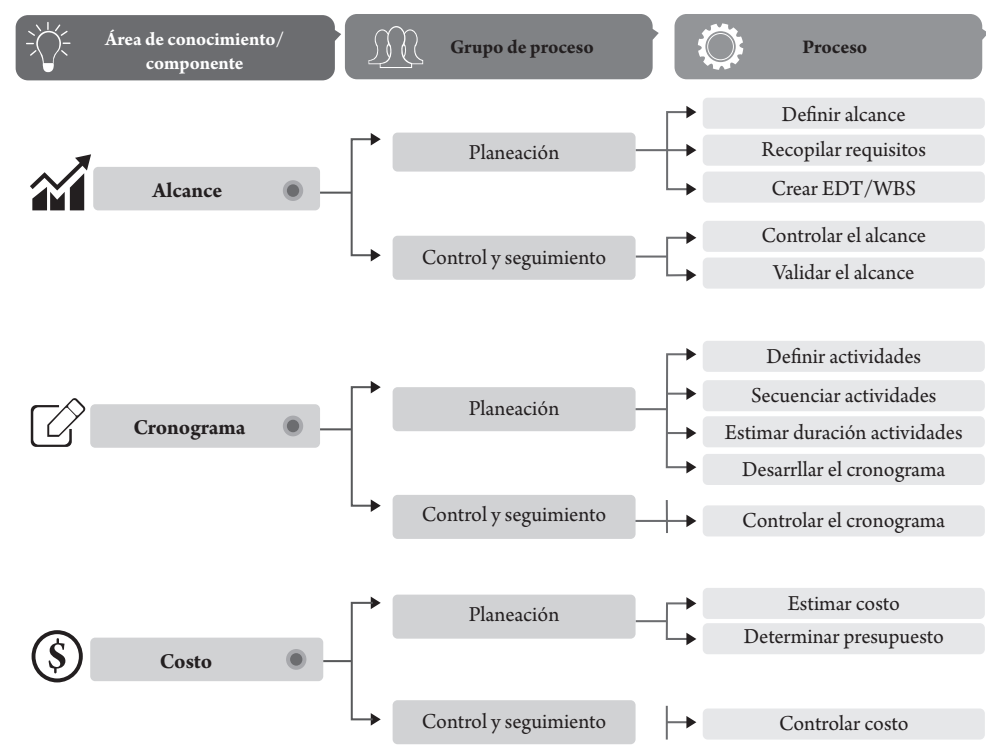

Fuente. PMI, 2017.

El PMBOK incluye diez áreas de conocimiento —integración, alcance, cronograma, costos, calidad, recursos, comunicaciones, riesgos, adquisiciones e interesados - y cinco grupos de procesos —inicio, planeación, ejecución, monitoreo y control y cierre-, de modo que se generaron 49 procesos. En la investigación se utilizaron solo tres áreas de conocimiento, denominadas componentes: alcance, cronograma y costo, con sus correspondientes grupos de procesos. Se trabajaron en 13 procesos de los 49 que recomienda el PMI. Para cada proceso del componente de estas áreas de conocimiento se diseñó una pregunta relacionada con su respectivo cumplimiento (véase la Tabla 1).

Tabla 1. Diseño del instrumento: preguntas por cada componente

\begin{tabular}{|c|c|c|c|}
\hline Grupo de proceso & $\begin{array}{c}\text { Preguntas del componente } \\
\text { alcance }\end{array}$ & $\begin{array}{c}\text { Preguntas del componente } \\
\text { cronograma }\end{array}$ & $\begin{array}{l}\text { Preguntas del componente } \\
\text { costo }\end{array}$ \\
\hline \multirow{4}{*}{ Planeación } & $\begin{array}{l}1 \text { ¿Cómo realiza la recopilación } \\
\text { de las características o } \\
\text { especificaciones de los resultados? }\end{array}$ & $\begin{array}{l}\text { 6. ¿De las siguientes } \\
\text { herramientas cuál es la } \\
\text { más utiliza para definir las } \\
\text { actividades del proyecto? }\end{array}$ & \multirow{2}{*}{$\begin{array}{l}\text { 12. ¿De las siguientes } \\
\text { herramientas cuál es la que } \\
\text { más desarrolla para determinar } \\
\text { los costos del proyecto? }\end{array}$} \\
\hline & $\begin{array}{l}\text { 2. ¿Qué condiciones desarrolla } \\
\text { para definir el alcance del } \\
\text { proyecto? }\end{array}$ & $\begin{array}{l}\text { 7. ¿De los siguientes procesos } \\
\text { cuál es el que más desarrolla para } \\
\text { identificar la secuencia de las } \\
\text { actividades? }\end{array}$ & \\
\hline & \multirow{2}{*}{$\begin{array}{l}\text { 3. ¿Qué procesos realiza con } \\
\text { respecto a la definición del trabajo } \\
\text { a desarrollar? }\end{array}$} & $\begin{array}{l}\text { 8. ¿De las siguientes } \\
\text { herramientas cuál es la que más } \\
\text { desarrolla para determinar la } \\
\text { duración de las actividades? }\end{array}$ & $\begin{array}{l}\text { 13. ¿De las siguientes } \\
\text { actividades cuál es la que } \\
\text { más desarrolla para definir el } \\
\text { presupuesto? }\end{array}$ \\
\hline & & $\begin{array}{l}\text { 9. ¿De las siguientes actividades } \\
\text { cuál es la que más desarrolla } \\
\text { para determinar el tiempo de un } \\
\text { proyecto? }\end{array}$ & \\
\hline
\end{tabular}




\begin{tabular}{|c|l|l|l|}
\hline \multirow{2}{*}{$\begin{array}{c}\text { Control y } \\
\text { seguimiento }\end{array}$} & $\begin{array}{l}\text { 4. ¿De las siguientes actividades } \\
\text { cuál es la que más desarrolla para } \\
\text { controlar el alcance? }\end{array}$ & $\begin{array}{l}10 . \text { ¿De las siguientes actividades } \\
\text { cuál es la más desarrolla para } \\
\text { controlar el estado del proyecto } \\
\text { en cuanto al tiempo? }\end{array}$ & $\begin{array}{l}\text { 14. ¿De las siguientes } \\
\text { actividades cuál es la que más } \\
\text { desarrolla para controlar el } \\
\text { estado del proyecto en cuanto } \\
\text { a la ejecución presupuestal? }\end{array}$ \\
\cline { 2 - 4 } & $\begin{array}{l}\text { 5. ¿De las siguientes actividades } \\
\text { cuál es la que más desarrolla } \\
\text { para formalizar la aceptación y la } \\
\text { validez? }\end{array}$ & $\begin{array}{l}\text { 11. Si la investigación presenta } \\
\text { retraso de entrega, icuál acción } \\
\text { es la que más aplica en sus } \\
\text { proyectos? }\end{array}$ & $\begin{array}{l}\text { 15. Si la investigación presenta } \\
\text { sobrecostos, icuál acción } \\
\text { es la que más aplica en sus } \\
\text { proyectos? }\end{array}$ \\
\hline
\end{tabular}

Fuente. Elaboración propia.

Para el cuestionario se diseñaron cuatro tipos de respuesta que se enlistan y describen a continuación.

1. Respuesta de forma gradual. Esta se da de acuerdo con la alta, media o baja aplicación de los lineamientos que propone el PMI en la guía $\mathrm{PMBOK}$ para cumplir con el proceso identificado, los cuales pueden ser más de dos opciones por pregunta. También implica aumentar la complejidad de los procesos, las herramientas, las técnicas o los métodos de una respuesta a otra.

2. Respuesta técnica del PMBOK. Es una opción de respuesta que corresponde a procesos, herramientas, técnicas o métodos que el PMBOK aplica para una correcta gerencia de proyectos; en términos de gradualidad, esta opción es la más compleja y completa.

3. Respuesta errónea o no cumplimiento. Implica una opción de respuesta de una mala práctica de gerencia o una nula práctica de gerencia por cada proceso.

4. Respuesta que se obtiene a partir de entrevistas con investigadores. Esta es la opción que con frecuencia utilizan los investigadores, como una práctica que ellos realizan para la gerencia de proyectos de investigación, desarrollo tecnológico e innovación.

Para la validación del cuestionario se realizó un juicio de expertos con cinco project management professionals (PMP), seleccionados por tener posgrados en gerencia de proyectos $y$ haber aplicado su experiencia en diferentes tipologías de proyectos. En el juicio de experto se revisó el cuestionario en términos técnicos: terminología, coherencia y pertinencia en gerencia de proyectos $y$ en los procesos del PMBOK. Además, calificaron cada una de las respuestas que se plantearon con el fin de diseñar el método de valoración en prácticas de gerencias.

\subsection{Método de valoración}

Para la valoración de las respuestas del cuestionario y cuantificar el índice del nivel de gerencia (ING) se diseña un método con su propia formulación matemática aplicable a un único cuestionario. Por medio del juicio de expertos se califica cada respuesta, se pondera cada pregunta y cada componente, según la relevancia en aplicación de prácticas en gerencia de proyectos.

En la calificación de las respuestas cada experto asigna un valor de 0 a 100 a la respuesta de cada pregunta, donde 0 es la respuesta más errónea y 100 la respuesta más acertada. Las opciones de respuestas no son excluyentes y se les pueden asignar valores iguales a respuestas de una misma pregunta.

Para la calificación de preguntas el experto asigna una ponderación dentro de un porcentaje de 100 a cada pregunta de un componente. Esta asignación representa la importancia de cada proceso dentro de cada componente evaluado en la gerencia de proyectos. Después se realiza una calificación de componentes, igualmente asignando una ponderación dentro de un porcentaje de 100, 
de modo que representa la importancia de cada componente - alcance, cronograma, costo - en la aplicación de prácticas de gerencia. La calificación tanto de cada pregunta de un componente como de los tres componentes dará una suma de $100 \%$.

\subsection{Población y muestra}

La población definida es de $1571^{5}$ grupos reconocidos y categorizados de la capital de Colombia. En el tamaño de la muestra se utilizó el muestreo estratificado para poblaciones finitas; se determina con niveles de confianza del $90 \%$ a $100 \%$ y error de $1 \%$ al $10 \%$ para la población total; para la selección de la muestra se utiliza un muestreo por cuotas.
De acuerdo con Pimienta-Lastra (2000), el muestreo por cuotas es una alternativa del muestreo probabilístico que mantiene una selección aleatoria y asegura empíricamente que cada miembro de la población tenga la misma probabilidad de ser parte de la muestra. A fin de mantener la aleatoriedad en la selección de la muestra, se elabora un marco muestral como referencia que permita identificar cada una de las características de la población, se envía el cuestionario a todos los grupos de la población y se logra una muestra de 329 grupos de investigación.

\section{Resultados}

ـespecificar y detallar el método para la valoración de prácticas en gerencia de proyectos en grupos de investigación, basado en el estándar del PMI (2017) — guía PMBOK-.

\subsection{Método para valorar la aplicación de prácticas en gerencia de proyectos de grupos de investigación}

Para la valoración de la aplicación de prácticas en gerencia de proyectos se cuantifican las respuestas obtenidas del cuestionario. El proceso de cuantificación se realiza por medio de la calificación del juicio de expertos, en busca de definir el ING para cada posible respuesta del cuestionario.

El indicador de nivel de gerencia (ING) es el valor cuantitativo que permite medir y comparar la aplicación de prácticas en gerencia de proyectos. Este valor se calcula para cada grupo de investigación y se determina una escala de alto, medio o bajo, según el puntaje obtenido. A fin de obtener el valor ING se realizan tres pasos: promedio de las calificaciones de los expertos en opciones de respuesta, ponderado de las preguntas dentro de los componentes y ponderado de los componentes.

El promedio de cada respuesta se calcula con la aplicación de la siguiente ecuación:

$$
L_{j k}=\frac{1}{n} \sum_{i=1}^{n} m_{i j k}
$$

En donde:

- $\quad k$ está asociado con el número de pregunta sometida a juicio de expertos, esto es, $k=$ $1,2,3,4,5,7,8,9,10,11,12,14,15$, en donde $k=$ preguntas 1 al 5 corresponden al componente alcance, $k=$ preguntas 6 al 11 corresponden al componente cronograma y $k=$ preguntas $12 \mathrm{al}$ 15 corresponden al componente costo.

- $\mathrm{m}_{i j k}$ es la calificación del experto entre 0 y $100 \mathrm{i}$, siendo $i$ el número de expertos, en la opción de respuesta $j$, de la pregunta $k$. 
- $n$ es el número de expertos - juicio de expertos compuesto por cinco profesionales de diferentes áreas de conocimiento con certificación de $\mathrm{PMP}$ - que calificaron, esto es, $n=5$.

- $\mathrm{L}_{j k}$ es el promedio de las calificaciones de los expertos en la opción de respuesta $j$ de la pregunta $k$.

El ponderado de las preguntas por componente se calcula con la aplicación de la siguiente ecuación:

$$
\begin{gathered}
C_{k l}=\frac{1}{5} \sum_{i=1}^{5} q_{i k l} \\
\text { Con } \sum_{k=1}^{K l} q_{i k l}=100
\end{gathered}
$$

En donde:

- $\quad l$ está asociado con el componente, esto es, $l=1$ (alcance), $l=2$ (cronograma) y $l=3$ (costo).

- $q_{i k l}$ es la calificación del experto $i$, a la pregunta $k$ en el componente $l$.

- $\quad n_{l}$ es el número de preguntas en el componente $l$, que son $n_{1}=5, n_{2}=6$ y $n_{3}=4$.

- $\mathrm{C}_{k l}$ es el peso que dan, en conjunto, los expertos a la pregunta $k$ dentro del componente $l$.
El ponderado de los componentes se calcula con la aplicación de la siguiente ecuación:

$$
B_{l}=\frac{1}{5} \sum_{i=1}^{n} r_{i l}
$$

$$
\text { Con } \sum_{i=1}^{i l} r_{i l}=100
$$

En donde:

- $r_{i l}$ es la calificación del experto $i$, a la componente $l$.

- $\quad B_{l}$ es el peso que dan, en conjunto, los expertos al componente $l$.

El cálculo del ING por respuesta se obtiene multiplicando (1), (2) y (3), expresado en la siguiente ecuación:

$$
I N G_{j k l}=L_{j \mathrm{k}} \times C_{k l} \times B_{l}
$$

Finalmente, se obtienen los valores cuantitativos

\begin{tabular}{|c|c|c|c|c|c|c|c|c|}
\hline \multicolumn{3}{|c|}{ Componente alcance } & \multicolumn{3}{|c|}{ Componente cronograma } & \multicolumn{3}{|c|}{ Componente costo } \\
\hline Pregunta & Respuesta & $I N G_{j k l}$ & Pregunta & Respuesta & $I N G_{i k l}$ & Pregunta & Respuesta & $I N G_{j k l}$ \\
\hline \multirow{5}{*}{1} & A & 5,8 & \multirow{5}{*}{6} & \multirow{2}{*}{ A } & \multirow{2}{*}{4} & \multirow{5}{*}{12} & \multirow{2}{*}{ A } & \multirow{2}{*}{6,7} \\
\hline & B & 7,1 & & & & & & \\
\hline & $\mathrm{C}$ & 6,4 & & $\mathrm{P}$ & . & & \multirow{2}{*}{ B } & \multirow{2}{*}{5,7} \\
\hline & $\mathrm{D}$ & 0 & & D & 0 & & & \\
\hline & $\mathrm{E}$ & 3,1 & & $\mathrm{C}$ & 2,1 & & $\mathrm{C}$ & 0 \\
\hline \multirow{6}{*}{2} & A & 1,4 & \multirow{6}{*}{7} & \multirow{2}{*}{ A } & \multirow{2}{*}{3,7} & \multirow{6}{*}{13} & \multirow{2}{*}{ A } & \multirow{2}{*}{3,2} \\
\hline & B & 5,5 & & & & & & \\
\hline & $\mathrm{C}$ & 7,5 & & B & 4,2 & & B & 4,4 \\
\hline & $\mathrm{D}$ & 6,6 & & \multirow{2}{*}{$\mathrm{C}$} & \multirow{2}{*}{0} & & $\mathrm{C}$ & 7,5 \\
\hline & $\mathrm{E}$ & 0 & & & & & $\mathrm{D}$ & 0 \\
\hline & $\mathrm{F}$ & 2,1 & & D & 2,2 & & $\mathrm{E}$ & 1,1 \\
\hline
\end{tabular}
finales - ING - para cada opción de respuesta en cada pregunta de los componentes alcance, cronograma y costo (véase la Tabla 2).

Tabla 2. Valores de ING por cada respuesta 


\begin{tabular}{|c|c|c|c|c|c|c|c|c|}
\hline \multirow{5}{*}{3} & A & 3,9 & \multirow{5}{*}{8} & \multirow{2}{*}{ A } & \multirow{2}{*}{4,1} & \multirow{5}{*}{14} & A & 4,1 \\
\hline & B & 8,5 & & & & & B & 7,3 \\
\hline & C & 7,9 & & \multirow{2}{*}{ B } & \multirow{2}{*}{3,4} & & $\mathrm{C}$ & 6,5 \\
\hline & $\mathrm{D}$ & 0 & & & & & $\mathrm{D}$ & 0 \\
\hline & E & 2,5 & & $\mathrm{C}$ & 0 & & E & 0,5 \\
\hline \multirow{6}{*}{4} & A & 3,8 & \multirow{6}{*}{9} & A & 1.7 & \multirow{6}{*}{15} & A & 0.2 \\
\hline & B & 7 & & B & 3.4 & & B & 2.2 \\
\hline & $\mathrm{C}$ & 7,3 & & $\mathrm{C}$ & 4.8 & & C & 1.3 \\
\hline & $\mathrm{D}$ & 0 & & $\mathrm{D}$ & 6,6 & & $\mathrm{D}$ & 2.9 \\
\hline & \multirow{2}{*}{ E } & \multirow{2}{*}{3,1} & & E & 0,1 & & E & 6.9 \\
\hline & & & & $\mathrm{F}$ & 0,7 & & $\mathrm{~F}$ & 0.9 \\
\hline \multirow{5}{*}{5} & A & 4,5 & \multirow{5}{*}{10} & A & 2,2 & & & \\
\hline & B & 5,6 & & B & 5,2 & & & \\
\hline & $\mathrm{C}$ & 4,5 & & C & 4,9 & & & \\
\hline & D & 0 & & D & 0 & & & \\
\hline & E & 3,9 & & E & 2,1 & & & \\
\hline & & & \multirow{6}{*}{11} & A & 0,8 & & & \\
\hline & & & & B & 1,3 & & & \\
\hline & & & & C & 1,6 & & & \\
\hline & & & & $\mathrm{D}$ & 1,6 & & & \\
\hline & & & & $\mathrm{E}$ & 4,1 & & & \\
\hline & & & & $\mathrm{F}$ & 0 & & & \\
\hline
\end{tabular}

Fuente. Elaboración propia.

Los valores de ING corresponden a la calificación consolidada por cada respuesta obtenida del juicio de expertos.

Las opciones de respuestas de todas las preguntas del cuestionario son condicionadas por ser de opción múltiple única respuesta. Es decir, al sumar las opciones de respuesta con menor valor de ING se obtiene un puntaje de 1,0, y al sumar las opciones de respuesta con mayor valor de ING se obtiene un puntaje de 93.

Los valores de ING de los grupos de investigación estarán entre 1,0 y 93 . Siendo 93 el máximo puntaje y 1,0 el mínimo puntaje para la aplicación de prácticas de gerencia.

\subsection{Aplicación del método de valoración de prácticas de gerencia de proyectos en grupos de investigación de Colombia}

Al aplicar el método de valoración a los 329 grupos de investigación identificados en la muestra se encontró que en el componente alcance el proceso que más grupos de investigación realizan es crear la estructura de desglose de trabajo ${ }^{6}$; con un ING de respuesta de 8,5 lo aplica el $44 \%$ de los grupos; mientras que el proceso que menos grupos realizan es validar el alcance, con solo el $4 \%$ de los grupos y un ING de 7,3. En la figura 2 se presentan los resultados obtenidos para los cinco procesos gerenciales de alcance.

6 La EDT es una descomposición jerárquica orientada al entregable, relativa al trabajo que será ejecutado por el equipo del proyecto para lograr los objetivos del proyecto y crear los entregables requeridos (Project Management Institute, 2017). 
Figura 2. Grupos de investigación que aplican los procesos e ING de las respuestas del componente alcance

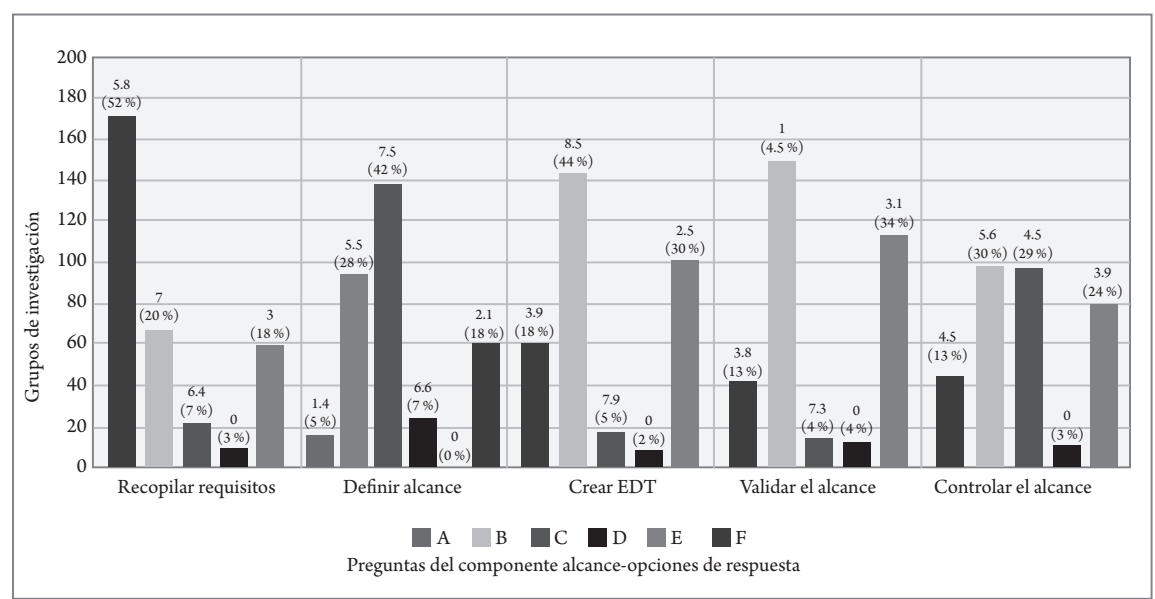

Fuente. Elaboración propia.

En la figura 2 se puede determinar la cantidad de grupos que seleccionaron la práctica de gerencia y utilizan por cada proceso del componente alcance los valores indicados sobre los porcentajes correspondientes a los valores de ING; los valores más altos de ING pertenecen a las prácticas de gerencia más convenientes para cada proceso.

Entre las mejores prácticas del componente cronograma, el proceso que más grupos de investigación realiza es controlar el cronograma con un ING de respuesta de 5,2, puesto que la aplica el $52 \%$ de los grupos; le sigue definir actividades con un ING de 2, y la aplica el $68 \%$ de los grupos; mientras que el proceso que menos grupos realizan es controlar el cronograma — con acciones correctivas-, con solo el 4 $\%$ de los grupos y un ING de 4,1. En la figura 3 se presentan los resultados obtenidos para los seis procesos gerenciales de cronograma.

Figura 3. Grupos de investigación que aplican los procesos e ING de las respuestas del componente cronograma

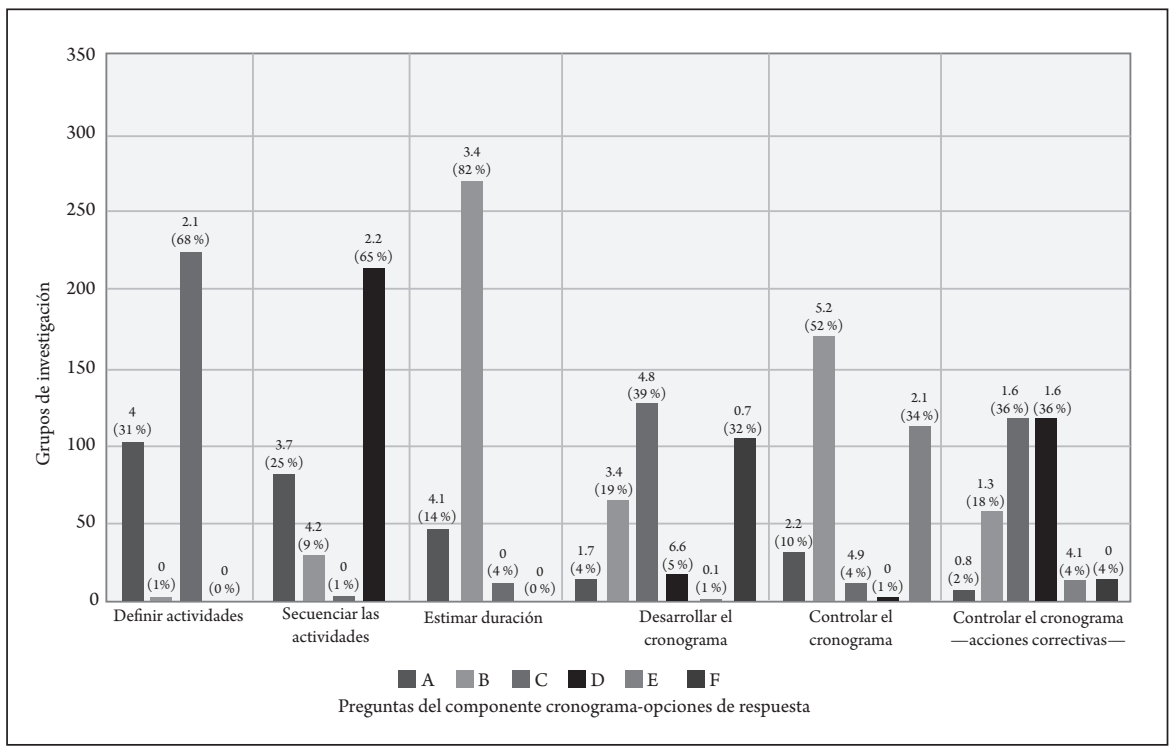

Fuente. Elaboración propia. 
En la figura 3 se puede determinar la cantidad de grupos que seleccionaron la práctica de gerencia que utilizan por cada proceso del componente cronograma, los valores indicados sobre los porcentajes corresponden a los valores de ING; los valores más altos de ING pertenecen a las prácticas de gerencia más convenientes para cada proceso.

Entre las mejores prácticas del componente costo, el proceso que más grupos de investigación realizan es controlar el costo, con un ING de respuesta de 7,3, pues lo aplica el $66 \%$ de los grupos, mientras que el proceso que menos grupos realizan es determinar el presupuesto, con solo el $4 \%$ de los grupos y un ING de 7,3. En la figura 4 se presentan los resultados obtenidos para los cuatro procesos gerenciales de costo.

Figura 4. Grupos de investigación que aplican los procesos e ING de las respuestas del componente costo

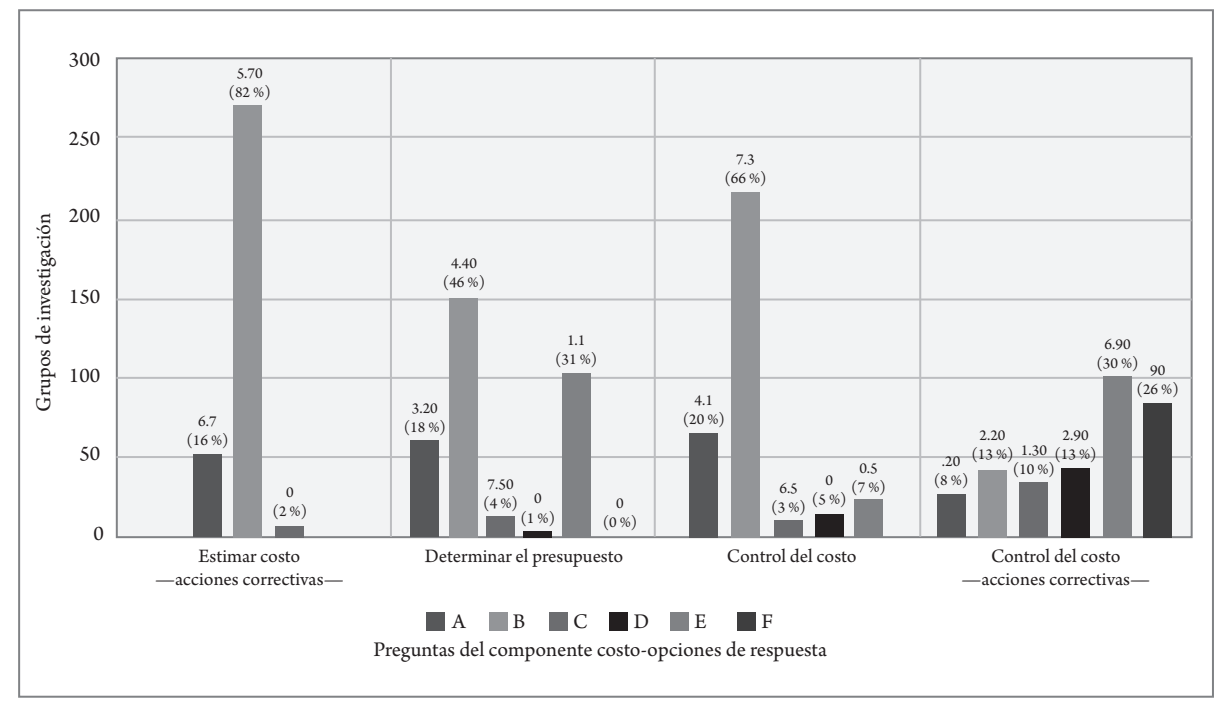

Fuente. Elaboración propia.

En la figura 4 Se puede determinar la cantidad de grupos que seleccionaron la práctica de gerencia y utilizan por cada proceso del componente costo; los valores indicados sobre los porcentajes corresponden a los valores de ING, mientras los valores más altos de ING pertenecen a las prácticas de gerencia más convenientes para cada proceso.

Las respuestas de los cuestionarios diligenciados por los grupos de investigación se valoran con el ING. La sumatoria de los ING por respuesta indica un nivel de gerencia por cada grupo de investigación. El intervalo de ING de los grupos de investigación se encuentra entre 17 y 84,8 .

En la tabla 3 se pueden identificar los niveles de gerencia de proyectos por cada una de las categorías ${ }^{7}$ de los grupos de investigación.

Categoría otorgada por Minciencias de acuerdo con la medición del grupo en temas de producción científica. 
Tabla 3. Valores de ING en los grupos de investigación

\begin{tabular}{|c|c|c|c|c|}
\hline ING & N & Promedio & Mínimo & Máximo \\
\hline Grupos & 329 & 61,4 & 17,0 & 84,8 \\
\hline Categoría A1 & 50 & 59,41 & 36.4 & 81,4 \\
\hline Categoría A & 64 & 65,15 & 38.9 & 84,4 \\
\hline Categoría B & 93 & 60,2 & 17,0 & 84,8 \\
\hline Categoría C & 122 & 61,12 & 29.2 & 82,0 \\
\hline
\end{tabular}

Fuente. Elaboración propia.

Las categorías en las tablas corresponden a niveles otorgados a los grupos de investigación, siendo A1 los grupos con mayor categoría en investigación y $\mathrm{C}$ aquellos con menor categoría.

Con cada valor de ING obtenido por los grupos de investigación se realizan histogramas a fin de poder observar que la mayoría de los grupos de investigación tienen un valor de gerencia entre 60 y 70 (véase la Figura 5).

Figura 5. Comportamiento del ING en los grupos de investigación

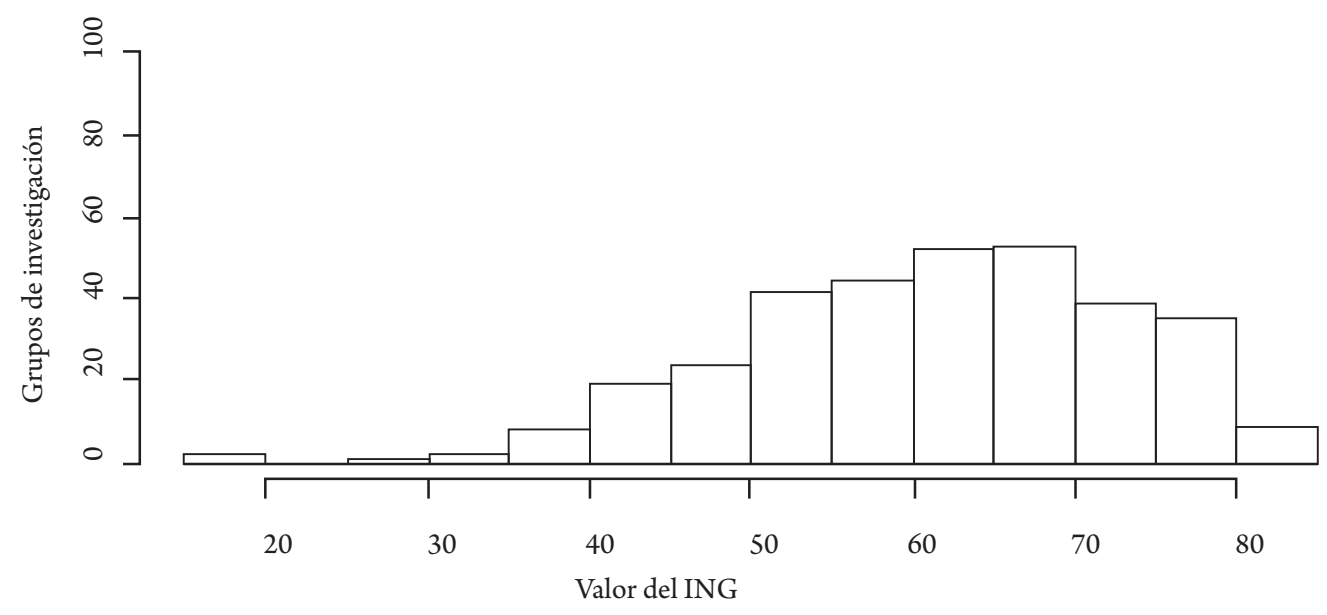

Fuente. Elaboración propia.

La concentración de los grupos de investigación se ubica entre los valores de 60 a 70 ING.

\subsection{Niveles de los grupos de investigación según sus prácticas en gerencia de proyectos}

Se establecieron tres niveles de aplicación de prácticas de gerencia — bajo, medio, alto-, para lo cual se utilizó el consenso de juicio de expertos, quienes consideraron que el nivel bajo se encuentra entre 0 y 36 , el nivel medio entre 37 y 69 y el nivel alto entre 70 y 100. De esta manera se realizó la distribución de los grupos de investigación en cada uno de los niveles (véase la Tabla 4). 
Tabla 4. Niveles de ING en los grupos de investigación

\begin{tabular}{|c|c|c|c|}
\hline Nivel & Bajo & Medio & Alto \\
\hline Medición ING & $0-36$ & $37-69$ & $70-100$ \\
\hline Grupos & 7 & 244 & 78 \\
\hline
\end{tabular}

Fuente. Elaboración propia.

En el nivel medio se encuentra el 74,1 \% de los grupos de la muestra, el 23,7 \% en el nivel alto y en el nivel bajo un porcentaje de $0,022 \%$.

\section{Discusión}

$\mathrm{C}$ on base en los resultados obtenidos se puede afirmar que la aplicación de prácticas en gerencia de proyectos de los grupos de investigación no se considera apropiada, pero tampoco se consideran malas prácticas; se evidencia que son prácticas fruto de la experiencia en formulación y gerencia de proyectos de $\mathrm{I}+\mathrm{D}+\mathrm{i}$ y que, como lo señalan Kuchta y Skowron (2015), son prácticas informales, por no existir directrices uniformes en gerencia de proyectos $\mathrm{I}+\mathrm{D}+\mathrm{i}$.

Para Youker (2002), en la gerencia de proyectos de $\mathrm{I}+\mathrm{D}+\mathrm{i}$ la calidad del proyecto tiene una mayor prioridad que otros componentes. Sin embargo, se identificó que un alto porcentaje de investigadores no cuenta con buenas prácticas para la identificación de requisitos. De hecho, la validación del alcance es el proceso que más porcentaje de investigadores no realiza dentro del componente alcance, de manera que son estos procesos directamente relacionados con la calidad de los resultados o productos de investigación.
En cuanto a la complejidad de los proyectos de I+D+i que señaló Morandi (2013), no se midió el desempeño de los proyectos referente a las prácticas de gerencia, pero sí se observa una baja profesionalización en la gerencia de este tipo de proyectos, al plantear hipótesis en cuanto a la complejidad y la baja profesionalización en la gerencia.

Es evidente que los investigadores realizan una correcta planeación del cronograma, pero presentan dificultades para realizar acciones correctivas en el control del cronograma, lo cual sustentan Goldstein y Kearney (2020), así como a Youker (2002), en el sentido de que los proyectos de $\mathrm{I}+\mathrm{D}+\mathrm{i}$ tienden a ser a largo plazo, de modo que modifican con periodicidad su frecuencia. 


\section{Conclusiones}

$E^{1}$ método de valoración diseñado permite identificar y medir a las organizaciones que realizan gerencia de proyectos de manera empírica, como son los grupos de investigación que, según los resultados, afirman que no cuentan con profesionales en gerencia y son los investigadores quienes planean y ejecutan los proyectos.

Aplicando un método de medición adaptado para investigadores que realizan gerencia de proyectos - no para gerentes de proyectos - los resultados varían, de modo que se obtiene un mayor nivel de gerencia cuando el método de recolección de datos se diseña específicamente para este tipo de población. Esta conclusión es evidente cuando se comparan los resultados de esta investigación y los de la que realizaron Cassaneli et al. (2009) en la aplicación del modelo de madurez de Kerzner en centros de investigación de Argentina, donde se categorizaron en un nivel de madurez con conocimiento regular - de una escala de cuatro niveles, siendo regular el segundo nivel-. Al aplicar el método de valoración diseñado en esta investigación, el $75 \%$ de los grupos se ubica con puntaciones de ING superiores a 53,51 y un valor promedio 61,4 - los valores de ING del juicio de expertos se ubican entre 1,0 y 93 , de modo que 93 es el máximo puntaje y 1,0 el mínimo puntaje para la aplicación de prácticas de gerencia de proyectos-.

Se identifica que los investigadores manejan conceptos básicos de gerencia y las prácticas que utilizan son empíricas y generadas por la experiencia. Por ejemplo, en la validación del alcance no sienten la necesidad de identificar procesos o herramientas que validen la aceptación por la contraparte, de los resultados y los productos intermedios y finales de la investigación. Entienden que la responsabilidad de validar es del investigador en el sentido de realizar el control de calidad de los resultados y productos para tener validez. $\mathrm{Si}$ bien es cierto que el investigador debe tener la capacidad de medir la calidad de sus resultados y productos, en las buenas prácticas de gerencia se recomienda realizar inspecciones por parte del cliente en productos finales e intermedios con el fin de obtener la aceptación formal de ellos.

Para definir las actividades, estimar la duración y los costos, los investigadores realizan reuniones con el equipo de investigación y revisan proyectos de $\mathrm{I}+\mathrm{D}+\mathrm{i}$ anteriores, para lo cual toman como base las prácticas empíricas y los resultados de la experiencia en la formulación de proyectos. De acuerdo con el juicio de expertos estas prácticas son válidas.

En términos generales, se observa que la mayoría de los grupos de investigación planea el alcance y el cronograma, es decir, se centran en definir las actividades necesarias para obtener los entregables identificados y el producto del proyecto de investigación, los procesos a los que más le dedican esfuerzo gerencial. Contrario a esto, los grupos no toman acciones correctivas cuando se presenta atrasos en el seguimiento y el control, lo que implica un incumplimiento en el cronograma del proyecto y un aumento en el costo.

Con respecto al componente costo, los grupos realizan mayor control y seguimiento en los recursos que obtienen para financiar el proyecto de investigación con respecto a crear una línea base de costos que determina cuánto y cuándo se debe incurrir en dichos costos. Para los investigadores el cumplimiento del alcance es definitivamente lo más crítico, pues dedican sus prácticas a la planeación del alcance, especialmente en desglosar la estructura de trabajo, de modo que superan de manera significativa aspectos financieros y de cronograma. En conclusión, los investigadores a la hora de tomar decisiones en sus proyectos priorizan el cumplimiento del alcance del proyecto por encima de los de cronograma o presupuesto. 
Finalmente, el comportamiento del indicador de nivel de gerencia se adecúa de manera general en todas las categorías de los grupos de investigación. Los valores máximos de ING se encuentran en todas las categorías e indican que en cada una de las categorías se encuentran grupos de investigación que aplican prácticas adecuadas de gerencia de proyectos. Sin embargo, los valores mínimos se ubican en la categoría C y B, y se sitúa en la categoría B el grupo con el menor valor de ING. Ahora, si se enfoca en los valores mínimos, el comportamiento del ING es relativo a la categoría y se ubica en los más bajos, en las categorías C y B. Por otra parte, si se enfoca en los valores promedios, no existe ninguna relación, ya que el comportamiento del ING cuenta con valores similares en las cuatro categorías.

\section{Referencias}

Bark, R. H.; Kragt, M. E.; Robson, B. J. (2016). Evaluating an interdisciplinary research project: lessons learned for organizations, researchers and funders. International Journal of Project Management, 34(8), 1449-1459. DOI: https://doi. org/10.1016/j.ijproman.2016.08.004

Bortagaray, I. (2016). Políticas de ciencia, tecnología, e innovación sustentable e inclusiva en América Latina. Montevideo: Unesco.

Brem, A.; Radziwon, A. (2017). Efficient triple helix collaboration fosteringlocal niche innovation projects. A case from Denmark. Technological Forecasting and Social Change, 123, 130-141. DOI: https://doi.org/10.1016/j.techfore.2017.01.002

Cassaneli, A. N.; Dondero, M.; Morán, J. I.; Álvarez, J. Larrosa, N. (2009). Gestión de proyectos de investigación científica y tecnológica, medición de la madurez. Ponencia presentada en el XIII Congreso Internacional de Ingeniería de Proyectos. Universidad de Extremadura; Asociación Española de Ingeniería de Proyectos (AEIPRO); International Proyect Management Association (IPMA). Badajoz, España). 8-10 de julio. DOI: https://doi.org/10.13140/RG.2.1.1248.1768

Cassanelli, A.; Guiridlian-Guarino, M. C.; Fernandez-Sánchez, G. (2014). Proyectos de $I+D$, caracterización del tipo de investigación y el rol de gerente de proyectos. Ponencia presentada en el V Congreso Iberoamericano de Ingeniería de Proyectos. Universidad Técnica Particular de Loja. Loja, Ecuador. 13-14 de noviembre. DOI: https://doi.org/10.13140/ RG.2.1.2427.8248
Colciencias. (2013). Serie de estudios y evaluaciones de Ciencia, Tecnología e Innovación. Recuperado de https://bit. ly/3AcGR3f

Forozandeh, M.; Teimoury, E.; Makui, A. (2019). A mathematical formulation of time-cost and reliability optimization for supply chain management in researchdevelopment projects. Rairo-Operations Research, 53(4), 1385-1406. DOI: https://doi.org/10.1051/ro/2018068

Goldstein, A. P.; Kearney, M. (2020). Know when to fold 'em: an empirical description of risk management in public research funding. Research Policy, 49(1), 103873 DOI: https://doi.org/10.1016/j.respol.2019.103873

Huang, H.; Jong, S. (2019). Public funding for science and the value of corporate $\mathrm{R} \& \mathrm{D}$ projects; evidence from project initiation and termination decisions in cell therapy. Journal of Management Studies, 56(5), 1000-1039. DOI: https:// doi.org/10.1111/joms.12423

Kuchta, D.; Gładysz, B.; Skowron, D.; Betta, J. (2017). R\&D projects in the science sector. $R$ and D Management, 47(1), 88-110. DOI: https://doi.org/10.1111/radm.12158

Kuchta, D.; Skowron, D. (2015). Classification of R \& D projects and selection of $\mathrm{R} \& \mathrm{D}$ project. RњD Management, 46(5), 831-841. DOI: https://doi.org/10.1111/ radm. 12112

Lledo, P.; Rivarola, G. (2007). Director de Proyecto. Estados Unidos: Pearson.

Martínez, M. E.; Páez, I. (2017). Orientaciones estratégicas de los investigadores en el campo de la administración: un estudio comparativo entre regiones y disciplinas. Cuadernos de Administración, 29(52), 83-114. DOI: https://doi. org/10.11144/Javeriana.cao29-52.oeic

Mêgnigbêto, E. (2018). Modelling the triple helix of universityindustry-government relationships with game theory: core, shapley value and nucleolus as indicators of synergy within an innovation system. Journal of Informetrics, 12(4), 11181132. DOI: https://doi.org/10.1016/j.joi.2018.09.005

Millán, A. R. (2017). Evaluación de herramientas para la gerencia de proyectos de construcción basados en los principios del PMI y la experiencia. Prospectiva, 15(2), 5159. DOI: https://doi.org/10.15665/rp.v15i2.746

Montes-Guerra, M.; Gimena-Ramos, F.; Díez-Silva, M. (2013). Estándares y metodologías: instrumentos esenciales para la aplicación de la dirección de proyectos. Revista de Tecnología, 12(2), 11-23. DOI: https://doi. org/10.18270/rt.v12i2.757

Morandi, V. (2013). The management of industry-university joint research projects: how do partners coordinate and control R\&D activities? Journal of Technology Transfer. 38(2), 69-92. DOI: https://doi.org/10.1007/s10961-011-9228-5 
Mulcahy, R. (2013). Preparación para el examen PMP. Minnesota: RMC Publications.

Organización para la Cooperación y el Desarrollo Económicos. (2007). Manual Oslo. Guía para la recogida e interpretación de datos sobre innovación. Madrid: Tragsa. DOI: https:// doi.org/10.1787/9789264065659-es

Organización para la Cooperación y el Desarrollo Económicos. (2018). Manual de Frascati 2015: guía para la recopilación y presentación de información sobre la investigación y el desarrollo experimental. París: OECD Publishing DOI: https://doi.org/10.1787/9789264310681-es

Pimienta-Lastra, R. (2000). Encuestas probabilísticas vs. no probabilísticas. Política y Cultura, (13), 263-276.

Project Management Institute. (2017). Guía de los fundamentos para la dirección de proyectos. Newtown Square: Project Management Institute.

Sánchez-Serna, A.; Martínez, A.; Del Río-Cortina, A. (2017). Criterios de selección metodológica en la gerencia de proyectos. Espacios, 38(58), 798-1015. Recuperado de https://bit.ly/3xpxJ9N

Sarpong, D.; AbdRazak, A.; Alexander, E.; Meissner, D. (2017). Organizing practices of university, industry and government that facilitate - or impede - the transition to a hybrid triple helix model of innovation. Technological Forecasting and Social Change, 123, 142-152. DOI: https:// doi.org/10.1016/j.techfore.2015.11.032

Solarte-Pazos, L.; Sánchez-Arias, L. F. (2014). Gerencia de proyectos y estrategia organizacional: el modelo de madurez en gestión de proyectos. Revista Innovar, 24(52), 5-18. DOI: https://doi.org/10.15446/innovar. v24n52.42502

Youker, R. (2002). The difference between different types of projects. Max Wideman's PM Wisdom. Recuperado de https://bit.ly/3isCo6O 